Please send trade news information and

illustrations to Arveen Bajaj at the BDJ, Nature

Publishing Group, The Macmillan Building,

4-6 Crinan Street, London N1 9XW.

Trade news is provided as a service to readers

using text and images from the manufacturer,

supplier or distributor and does not imply

endorsement by the BDJ. Normal and prudent

research should be exercised before purchase or

use of any product mentioned.

\section{New testing technology}

The StatSpin 30 is a dedicated primary tube centrifuge that provides platelet rich plasma in 30 seconds. It is designed to remove red and white cells from the plasma and leave the platelets in the plasma. The plasma can then be used for tests that require platelet rich plasma.

The short spin minimises any heat build up and is simple to use. The StatSpin 30 spins four standard $13 \times 75 \mathrm{~mm}$ tubes, is small, quiet and takes very little bench space. Tube inserts are available for smaller tubes and a larger model is available upon request for larger volume tubes.

Reader response number 51

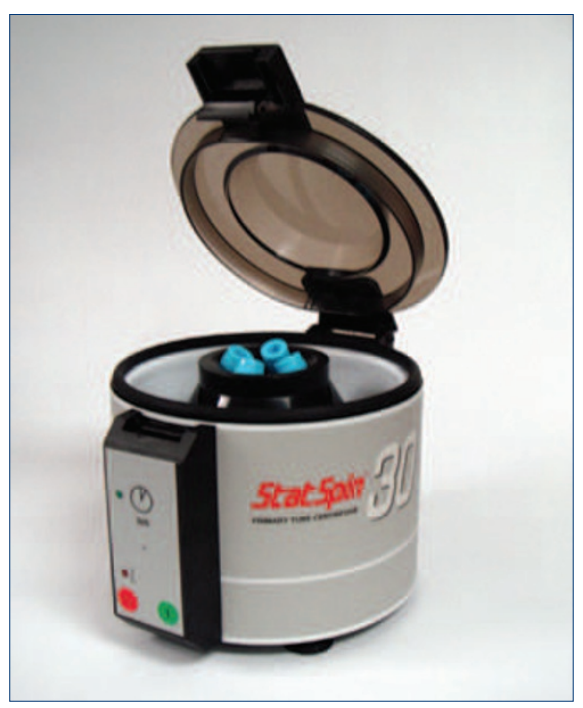

\section{Edge-to-edge clarity}

The range of Carl Zeiss magnification and illumination systems available from Nuview can offer many practical solutions and visual clarity during intricate medical procedures, without compromising comfort or movement, its makers claim.

The binocular magnifying devices allow edge-to-edge clarity of the magnified field of view while during long procedures, the loupes can be worn in two ways to ensure maximum comfort. They are available in nine different working

\section{Easy guide brushes}

Dentaid has responded to dental professional requests for a smaller and larger brush to complete its popular Interprox plus range. New Super Micro, distinguishable by its orange handle, has a wire diameter of $0.4 \mathrm{~mm}$. Interprox Plus Maxi is now the largest brush, with a red handle and $0.9 \mathrm{~mm}$ core.

The brushes have longer handles than traditional hand held brushes making them easy to guide around the mouth and easier to use for patients who struggle to manipulate short brushes.

The brush head is fixed at a predetermined angle alleviating the need for manual adjustment before use. The set angle of the brush ensures quick and easy access between teeth. Durable, half black, half white, bristle indicators are designed to help patients clean

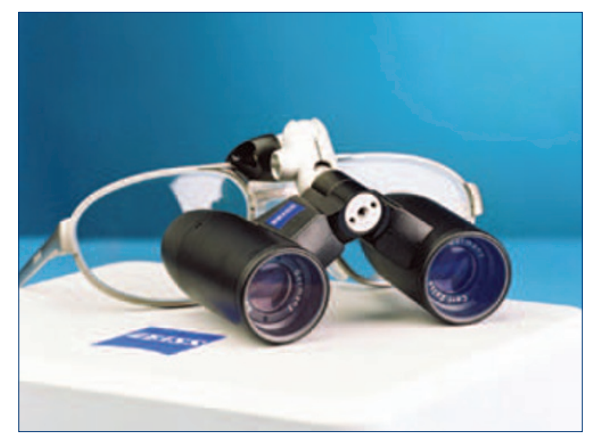

distances and in a choice of 11 different magnifications, from x3.2 to x8.

Reader response number 50

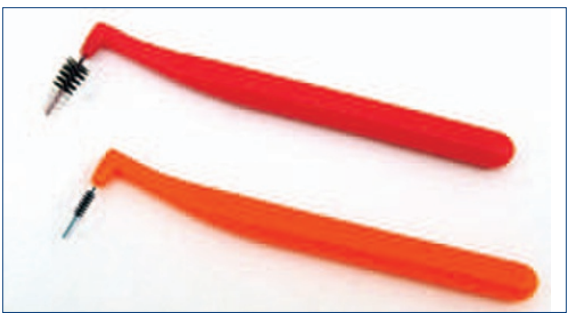

thoroughly by highlighting plaque and bleeding areas.

The enhanced choice of five sizes Super Micro, Micro, Mini, Conical and Maxi - are available from Dent-0-Care Ltd and other national suppliers in blister packs of six and boxes of 100 wrapped singles. Every brush has its own protective cap. For a free sample of Super Micro (orange) and Maxi (red) and enquiries about the range email kproven@dentocare.co.uk.

Reader response number 52

\title{
Metal ceramic combination
}

Encore! is a nickel-free, metal-lined ceramic bracket system that offers the aesthetics of ceramics with the functionality of metal, thus appealing to patients and clinicians alike.

The metal-lined archwire slot improves the sliding mechanics and can withstand higher torquing forces. Clinicians will also find the contoured base provides an ideal bracket fit whilst the tie wing area allows for easy ligation (the contours of the tiewing also improve patient comfort).

Each bracket has a removable colour coded ID dot to assist with selection and features dove tail grooves, which provide a firmer mechanical lock. Encore! forms part of the extensive range of Ortho Technology orthodontic products, exclusively available from The Dental Directory.

Reader response number 53

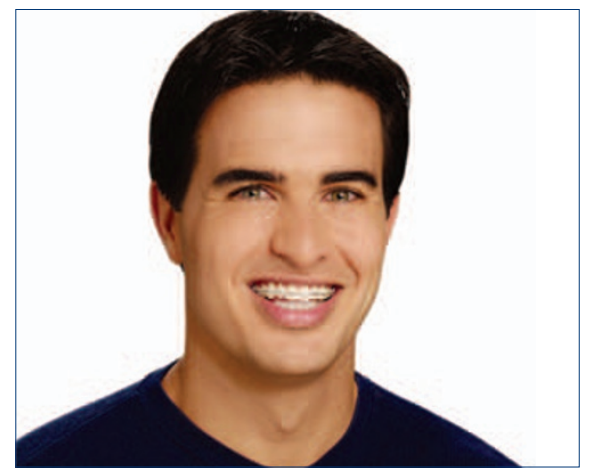




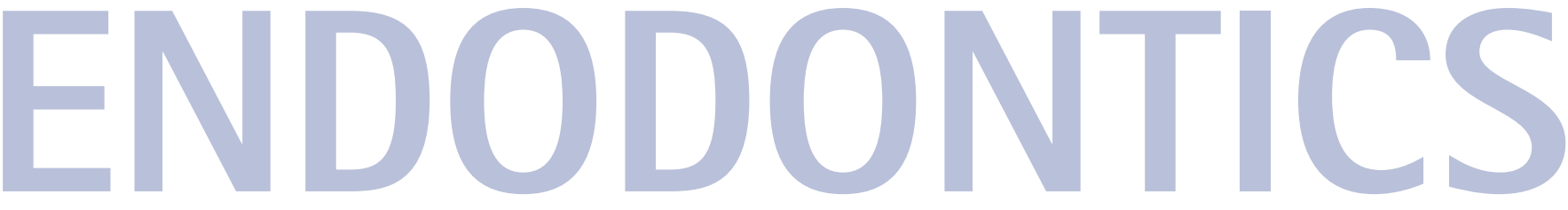

\section{Safe removal of fractured instruments}

The latest introductions to the range of Sendoline endodontic products from The Dental Directory are the Gutta Percha Remover Intro Kit, a File Extractor and Individual Endogrips.

The new gutta percha remover system will remove old filling material from the root canal and the instruments are used with the 'crown down' technique. The File Extractor has a unique and flexible spiral to facilitate easy and safe removal of fractured instruments in straight or bent canals. Users simply loosen the fragment, insert the extractor into the root canal, twist it $180^{\circ}$ clockwise, the spring then opens and can be pulled over the instrument; the extractor is then twisted anti-clockwise with a pulling motion to slowly remove the fragment.

Endogrip is a sleeve made of sterilisable silicone rubber, giving files a thicker handle making them ideal for use with gloves.

For the purpose of identification it is coded with four different colours to represent the different file types.

Reader response number 55

\section{All-round endo kit}

The limited edition ProEndo Discovery Kit from Dentsply, is an endo kit that includes materials and equipment required for cavity access, rotary root canal treatment including the X-Smart motor with two contra angles, obturation and re-treatment and accessory products. This kit is available to purchase until the end of March 2006.

Reader response number 54

\section{High adhesion to dentine}

The AH Plus is a two component paste-paste sealer from Dentsply. AH Plus has high adhesion to dentine in root canals, even without additional application of a bonding agent, giving rise to a tight seal.

It has a working time of four hours and the setting time is not adversely affected during thermoplastic obturation, making it suitable for use with warm obturation techniques such as Thermafil.

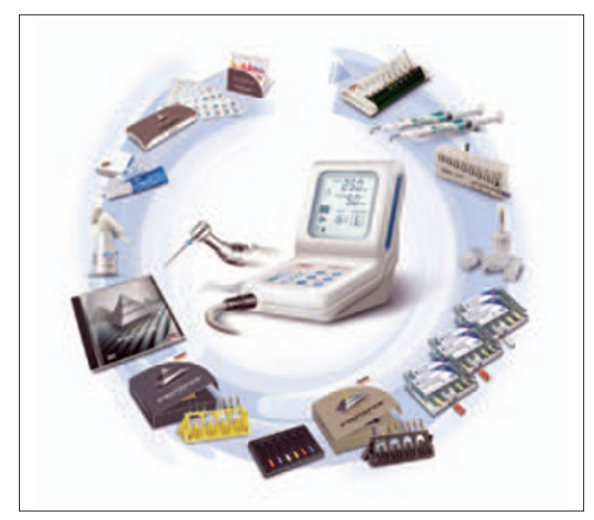

In addition AH Plus has high radiopacity and exhibits moderate anti-microbial properties giving it the potential to reduce the germ load in the canal significantly. It is now available in a new double-barrelled mixing syringe allowing for quick and direct dispensing with an optimal consistency.

Reader response number 56

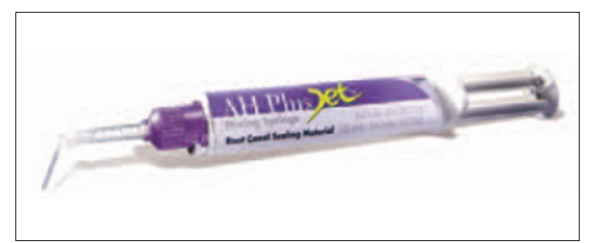

\section{Fluoride releasing core build-up material}

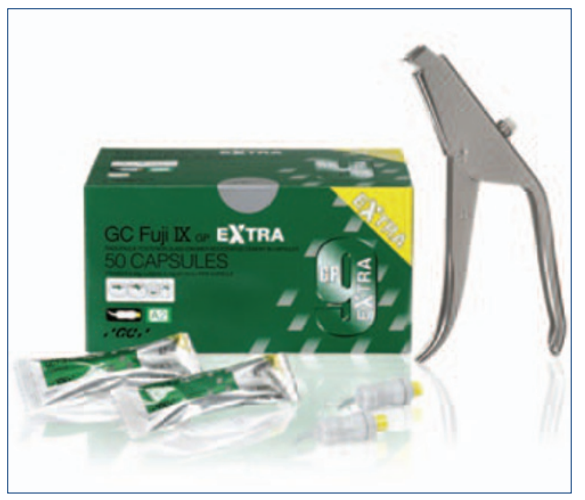

Fuji IX GP glass ionomer from GC (UK) Ltd is a core build-up material which can be used following endodontic treatment. The product is mixed to a putty-like consistency, either by hand or by using GC's capsulated system. The non-sticky material will not pull back when placed using metal hand instruments and due to its non-slump characteristics, it will stay exactly where you place it.

Fuji IX GP can be packed and shaped during the setting phase. This fluoride releasing material sets in four minutes and can be trimmed and finished immediately with water-cooled instruments. It will adhere to any pins or posts present without the need for additional bonding agents and exhibits high bond strengths to tooth structure.

It can be used in a variety of applications including permanent and semipermanent restorative work for children including the sealing of pits and fissures and as a geriatric restorative material, particularly where root caries are present.

Reader response number 57 


\section{Single-step cement}

New RelyX Fiber Posts and RelyX Unicem self-adhesive resin cement from $3 \mathrm{M}$ ESPE is a single-step cement which is especially useful for the cementation of endodontic posts as it requires no pretreatment of the root canal.

3M ESPE have also developed a system consisting of endodontic posts and drills in three sizes and specially designed elongated application tips for RelyX Unicem. RelyX fiber posts, RelyX fiber post drills and RelyX Unicem Aplicap elongation tips permit a safe and sim-

\section{Complete curing}

Premier Compcore from Myerson is a dual-cure, fluoride releasing, radiopaque core build-up material. The patented hyperbalanced molecular structure of Compcore ensures a dense cross-linking of the composite monomers to ensure complete curing all the way through the material.

This complete polymerisation significantly reduces shrinkage and contraction stresses that would inevitably lead to micro-leakage resulting in secondary caries. The low solubility ensures a perfect marginal seal.

The high compressive and tensile strengths of Compcore provide a solid foundation for final restorations. It is ple post cementation with RelyX Unicem self-adhesive resin cement.

Without pre-treatment of the root canal or of the post itself, the RelyX fiber post can be adhesively cemented in one step with no need to etch, prime, bond or silanize posts. The elongation tip makes it quick and easy to apply cement into the root canal from bottom to top. The elasticity of the material is similar to natural dentine, therefore the risk of root fracture is lower than with rigid metal or ceramic posts.

Reader response number 58

supplied in a convenient and compact automix syringe with both small tips and right-angled dispensing tips, allowing fast and precise placement. There are three shades to choose from; blue, A3 and white.

Reader response number 59

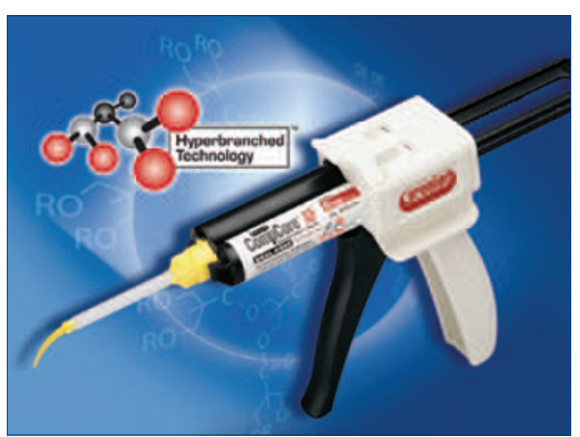

Extracting roots

made easier

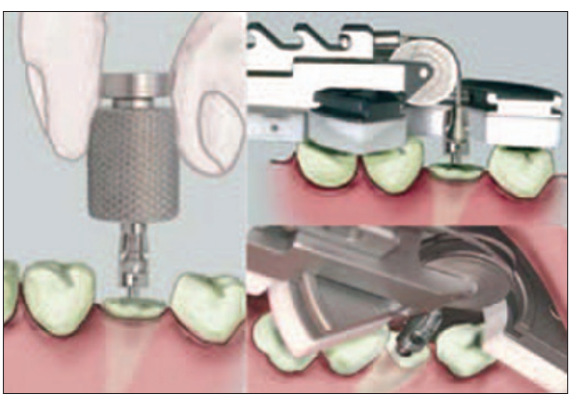

Benex-Control root extraction system from Meisinger is suited for the extraction of roots and root fragments in situations where conventional extraction using forceps would no longer be possible.

Due to the construction of the extractor, the root can be removed very easily in an extremely controlled way without causing injury to the bone.

Reader response number 62

\section{Controlled aspiration}

The Rotor Syringe range from Blackwell Supplies is designed to eliminate the use of barbs. The syringe uses the elasticity of the cartridge and thumb disk to induce carefully and easily controlled aspiration and can also be used with the Astra Self-Aspirating Cartridge. This method uses the unique design of the Astra Cartridge to induce aspiration.

The moulded components are made from Peek, offering a lightweight well balanced syringe. It also ensures that components will not rust after repeated exposure to autoclaving or chemicals commonly used within the dental surgery.

New to the range is the $1.8 \mathrm{ml}$ Rotor S/A (self-aspirating) syringe, which has the same construction and benefits of the $2.2 \mathrm{ml}$, but will accept the $1.8 \mathrm{ml}$ anaesthetic cartridges entering the UK dental market. Also the imperial thread on the Rotor $1.8 \mathrm{ml}$ syringe will accept the imperial thread hub needles currently sold throughout the UK and Ireland.

Straumann's single patient drills are supplied in a sterile pack complete with a specially designed integral handling piece. They are available in three different sets all with the same dimensions. Drill sharpness and optimal cutting properties means they are suitable for repeated single-patient use and are colorcoded to simplify the surgical sequence. Helping to combat the issues of infection control, the product represents a safe and simplified treatment solution.

Reader response number 61
Reader response number 63

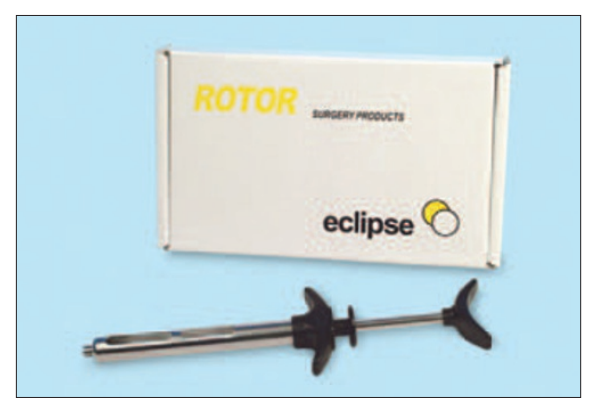

